\title{
Influence of guest lattice solvents on nanomechanical properties of pharmaceutical crystalline solids
}

\author{
Subhrajyoti Bhandary ${ }^{1,2}$, Pradip Kumar Mondal ${ }^{2}$, S. R. N. Kiran Mangalampalli ${ }^{3}$, Upadrasta Ramamurty ${ }^{4}$ and \\ Deepak Chopra ${ }^{2}$ \\ ${ }^{I}$ Department of Chemistry, Ghent University, Belgium, \\ ${ }^{2}$ Department of Chemistry, Indian Institute of Science Education and Research Bhopal, India, \\ ${ }^{3}$ Department of Physics and Nanotechnology, SRM Institute of Science and Technology, Kattankulathur, India, \\ ${ }^{4}$ School of Mechanical and Aerospace Engineering, Nanyang Technological University, Singapore. \\ subhrajyoti.bhandary@ugent.be
}

Solvates are ubiquitous in pharmaceutical crystalline solids [1]. Such solvates of a given drug molecule can exhibit different physicochemical properties such as solubility, thermal stability, and mechanical strength [2]. Hence, it is of extreme importance to understand the role of solvent molecules in determining the mechanical properties of the solvates via the nanoindentation technique, especially in view of the manufacturing of pharmaceutical tablets as well as the recent understanding of structure-property correlations in molecular crystals [3].

The current investigation reveals the role of guest lattice solvents in tailoring nanomechanical responses (hardness and elastic modulus) in different crystalline forms of two pharmaceutically active compounds, in particular with respect to the supramolecular structure and energetics of interaction topology of molecules. The nanomechanical responses of two crystalline phases of a dihydropyrimidine analogue are similar irrespective of the presence (or absence) of the guest dichloromethane lattice solvent [4]. In contrast, the mechanical properties of two differently solvated forms (acetonitrile and DMSO) of the second related compound are anisotropic (Fig. 1). The structural features of all crystalline forms demonstrate that depending on the presence of specific guest solvent molecule and interaction topology of host-guest intermolecular interactions along with their relative orientations in the crystal lattice, majorly decide their nanomechanical properties (hardness and elastic modulus).

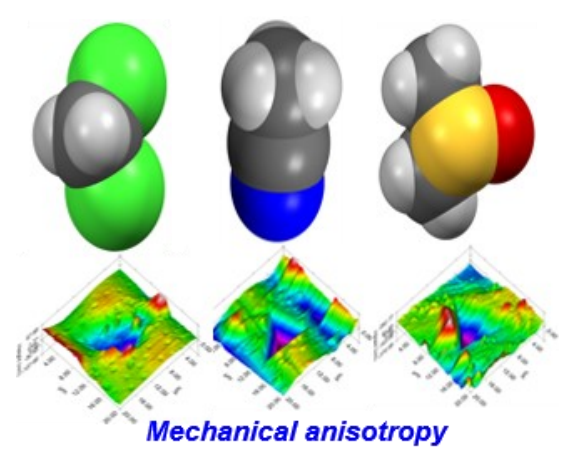

Figure 1. Guest solvent-dependence of nanomechanical properties in pharmaceutical crystalline solids.

[1] Griesser, U. J. (2006). The importance of solvates. In Polymorphism in the Pharmaceutical Industry (Ed.: R. Hilfiker), Wiley-VCH: Germany, pp. 211-257.

[2] Brittain, H. G. (2009). Polymorphism in Pharmaceutical Solids, Informa Health-care, NewYork.

[3] Wang, C. \& Sun, C. C. (2020). CrystEngComm 22, 1149.

[4] Bhandary, S., Rani, G., Mangalampalli, S. R. N. K., Rao, G. B. D., Ramamurty, U and Chopra, D. (2019). Chem. Asian J. $14,607$.

Keywords: pharmaceutical solvates; nanoindentation; mechanical properties and interaction topology 